\title{
DESIGNING SERVICES IN PANDEMIC CONTEXT
}

\author{
Rita Assoreira ALMENDRA \\ Universidade de Lisboa, Faculdade de Arquitetura, CIAUD, Portugal
}

\begin{abstract}
This paper addresses the challenge posed by COVID 19 to 60 bachelor students from the $3^{\text {rd }}$ year of the Design Programme at the Faculty of Architecture, University of Lisbon, that had to develop design services (in groups of 3/4 students) without the possibility of doing fieldwork or any other type of presential interaction. The imposed lockdown due to Coronavirus dictated not only changes on the themes and problems to be tackled but also introduced the need to adopt new processes and tools to collect, discuss and interpret information.

By the end of the course, 13 projects were developed (but not implemented) and 13 promotional videos were created to disseminate the work done. The projects were mostly focused on developing services to support old people, high education students, risk communities considering the adverse consequences of the virus in daily life and the wellbeing of people. Besides mentioning the main target groups, it is also important to refer to the areas of intervention that were chosen: mobility, education, security, health.

The critical analysis of the design processes as well as of the results of the work done in this design services' course allowed us to suggest numerous guidelines on how to collaborate in an effective way in design processes in a pandemic context that will hopefully help the academic community as well as students to better frame and act in this type of impacting situations.
\end{abstract}

\section{Keywords: Design high education, designing services, pandemic context}

\section{INTRODUCTION}

Designing services, as defined by the author and in terms of the construct to be used by students, means to design the relationship and connections among people, and amid people and objects having as the main goal the development of sustainable and ethically compromised solutions that will enhance people's wellbeing.

Designing services is also to operate mostly in the immaterial world depending heavily on people's behaviour. Thus, it must encompass a strong dialogue and empathy from the very beginning with those who will interact and be served by those services. That empathic and dialoguing process was severely constrained due to the pandemics and the interactions to be made had to occur almost without the physical presence/contact. The challenge we were facing is according to Bozkurt and Sharma [1] emergency remote teaching (ERT) and aims to maintain continuity of teaching-learning processes allowing students to complete the semester, even if the course was not what was originally planned. In fact, and a broader and inclusive term we are talking about Distance Education, which implies: a separation either geographical or in time between students and teachers, the existence of a two-way communication system among them, and the integration of education institutions [2]. The first and most critical aspect related to a teaching-learning process that was identified was: Interaction. Student-teacher and student-student interactions are the basis of the teaching-learning process and assume different typologies - individual discussions; design reviews; answer and questions moments; progress assessment; final assessment - that includes auto-evaluation and peer-evaluations besides the one of the teachers. In Design services that depend heavily on fieldwork interactions (in class; outside class) were assumed as the core aspect to be solved. 


\section{A FIELD WORK RESTRAINED BY COVID}

Going to the field to understand the context and be able to characterize it is a task to be developed with high intensity in the first phase of the development of a service. That was blocked due to the lockdown and implied new ways of gathering information, different modes of interaction, and engagement with people and their environment. This "revolution" had place at the beginning of the course and the students (ages ranging from 20 to 24) were severely impacted (both in psychological terms and in performance ones) since they never experienced such a restrained environment. Thus, the solution was to identify in which ways it was possible to pursue information and interaction without the presence and face-to-face contact. The immediate solution was to make intensive use of digital processes with a close follow-up from the teacher and opening and close class sessions' talks to stimulate positive attitudes and to discuss their difficulties and offer support and solutions. Regarding the practical aspects of the class processes especially the ones related to fieldwork, the challenge is well-posed by Favilla and Pita [3] when they ask "Are digital methods a possibility for opening the field, or are these methodologies limited in some cases? Can the proximity and collaboration generated by dialogue and interpersonal interaction be maintained in virtual contexts?"

Regarding these questions, it is important to notice that among the advantages several researchers identify in online modalities is the access to a large amount of information and the diminishment of barriers to participation, while in contrast there are the disadvantages of the lack of social interaction and the observed tendency that these modalities are more suitable to students with consistent and independent motivation and learning skills [4]. In special the disadvantages are intimately related to the essence of fieldwork.

\section{PROJECT BRIEFING AND THE PROPOSED PROCESS}

As previously said developing the process of designing services while being in a virtual environment was one of the consequences of the lockdown imposed by the first wave of COVID 19 (March/May 2020). That occurred without any previous preparation hence requiring on the part of teachers and students to find ways of collaborating and achieving results without jeopardizing its quality.

The Service design briefing (prepared in/to a Covid free moment) encouraged students to develop services having as a support element to their choices a table with different areas of intervention, target groups, and a set of possible stakeholders (from which they had to guarantee the participation of three). The choices to be made should have a clear impact in terms of social empowerment and sustainable commitment. In Table 1 we present the names of the projects as well as the areas of intervention and the target groups.

Table 1. Groups and its services thematic/target groups

\begin{tabular}{|c|c|c|c|}
\hline $\mathbf{N}^{\mathbf{o}}$ & Title service & Area of intervention & Target population \\
\hline 1 & Security at night - old town neighbourhoods & Security & Night users \\
\hline 2 & Giving the hand - living the city & Wellbeing & Informal caregivers \\
\hline 3 & Ajuda's Hand & Food waste & Vulnerable people \\
\hline 4 & Your Wave & School Dropout & Teenagers \\
\hline 5 & New Living & Elderly's Isolation & Elderly \\
\hline 6 & ReAjuda & Recycling & Poor neighbourhoods \\
\hline 7 & Sol a Lés - Solidary Transportation & Mobility & Elderly \\
\hline 8 & Happy Place & Temporary lodging & Students lodging \\
\hline 9 & Javô & Elderly's isolation & Elderly \\
\hline 10 & PorLisboa & Inclusive tourism & Disabled people \\
\hline 11 & Rehabita & Rehabilitation & urban citizens \\
\hline 12 & Overcoming Covid & Wellbeing & urban citizens \\
\hline 13 & Between Us & Education & University students \\
\hline
\end{tabular}

The definition of the problems to be tackled is the first and decisive step of the process in its exploratory phase and it includes a reflection about the causes and effects (consequences) of that problem. In Design services, the focus is the person and its interactions with other persons in a way that problems are solved in an ethical, inclusive, and sustainable way improving the wellbeing of all. 
As it can be seen in Figure 1 one of the techniques used to define the problem is the "Problem Tree" that must be articulated with the tool " 5 why?" (a cascade of 5 questions aiming at understanding the pertinence of the chosen problem) to establish a consistent and relevant problem to work with. This type of exploratory work was developed online thru the "zoom platform", using the breakout rooms so each group could discuss its ideas and jointly achieve the problem they would work with. The schemes, diagrams, and all the information gathering, analysis, and synthesis regarding problem identification were shared by the students that were using simultaneously "miro" - a collaboration tool that allows participants to ideate, diagram, map, and plan in real-time.

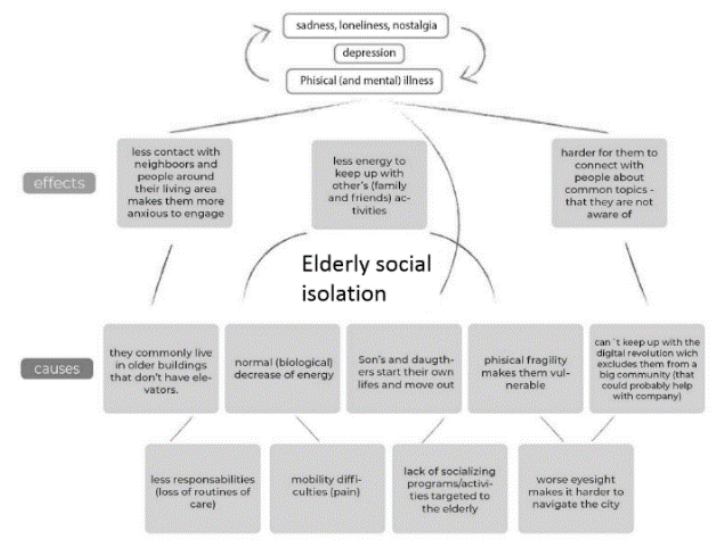

Figure 1. Example of "Problem Tree" definition - group 5

The making sense phase, which can be seen as an iteration of the exploratory one is a bridging phase towards a generative one in which students propose design services solutions to the identified problem. It is natural to do work on both phases, making sense and generation as a natural sequence or even simultaneously as co-evolving phases. These specific phases are supported by a set of activities that integrate the fieldwork. The process of making sense of the information gathered in the previous phase includes the use of tools such as 1) story world (allowing the students to define a survey to capture information about the user); 2) personas - designed with the information gathered in the 3) surveys; 4) storyboard - that implies the design of the whole process of interaction between user and service - either in a daily basis, week, month or even year basis (a kind of service journey) (see Figure 2).

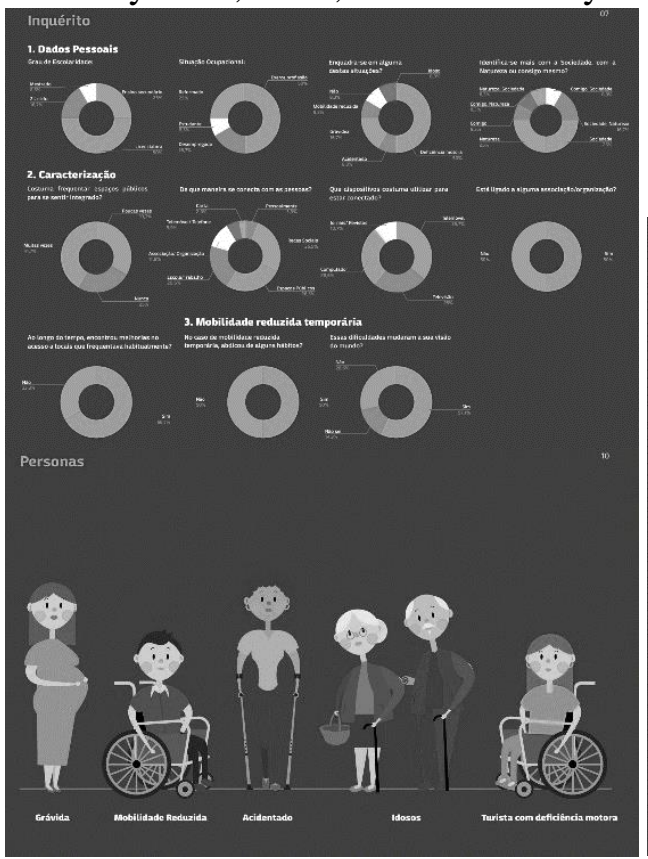

\section{Group 10 - Survey graphs - on the up-left side; Personas} image on the down left side; Storyboard excerpt - right side

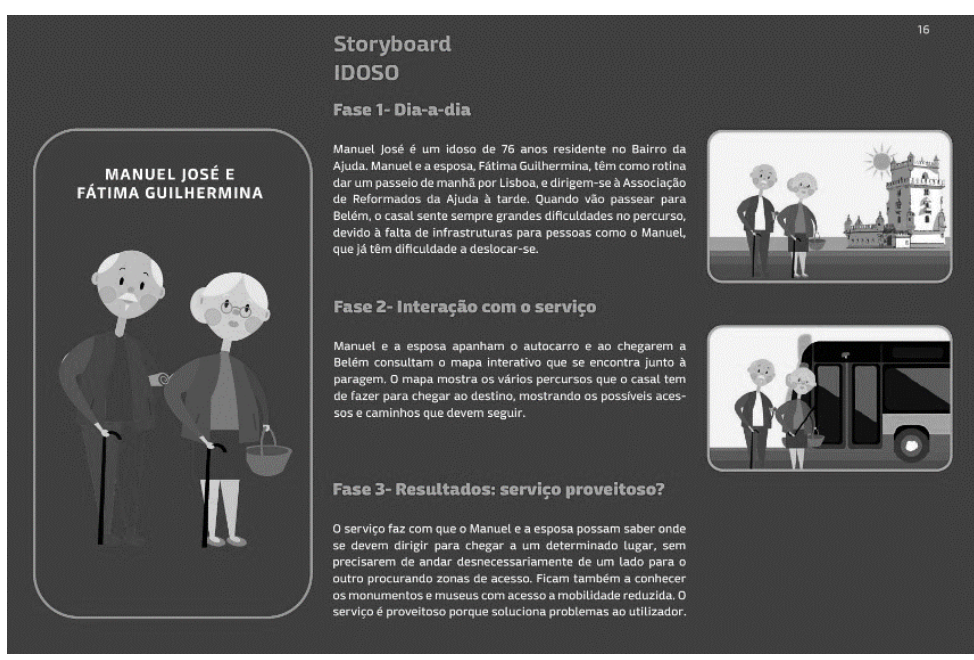

Figure 2. Example of "survey treatment"; "personas" and a "storyboard excerpt "from group 10 
Moreover, the generation of solutions depends on 5) the identification of all stakeholders that must be done by mapping them and defining a partner constellation in which the level of participation, the connection, and trade-offs among parts must be accurately defined; 6) the creation of a service proposition, (including its Logo symbol based in the 7) design of a service ecology and in the establishment of an 8) blueprint and of the 9) touchpoint display (see Figure 3). Besides the support of the teacher, students had the book of Kimbell and Julier [5] as a Guide to methods' usage and application.
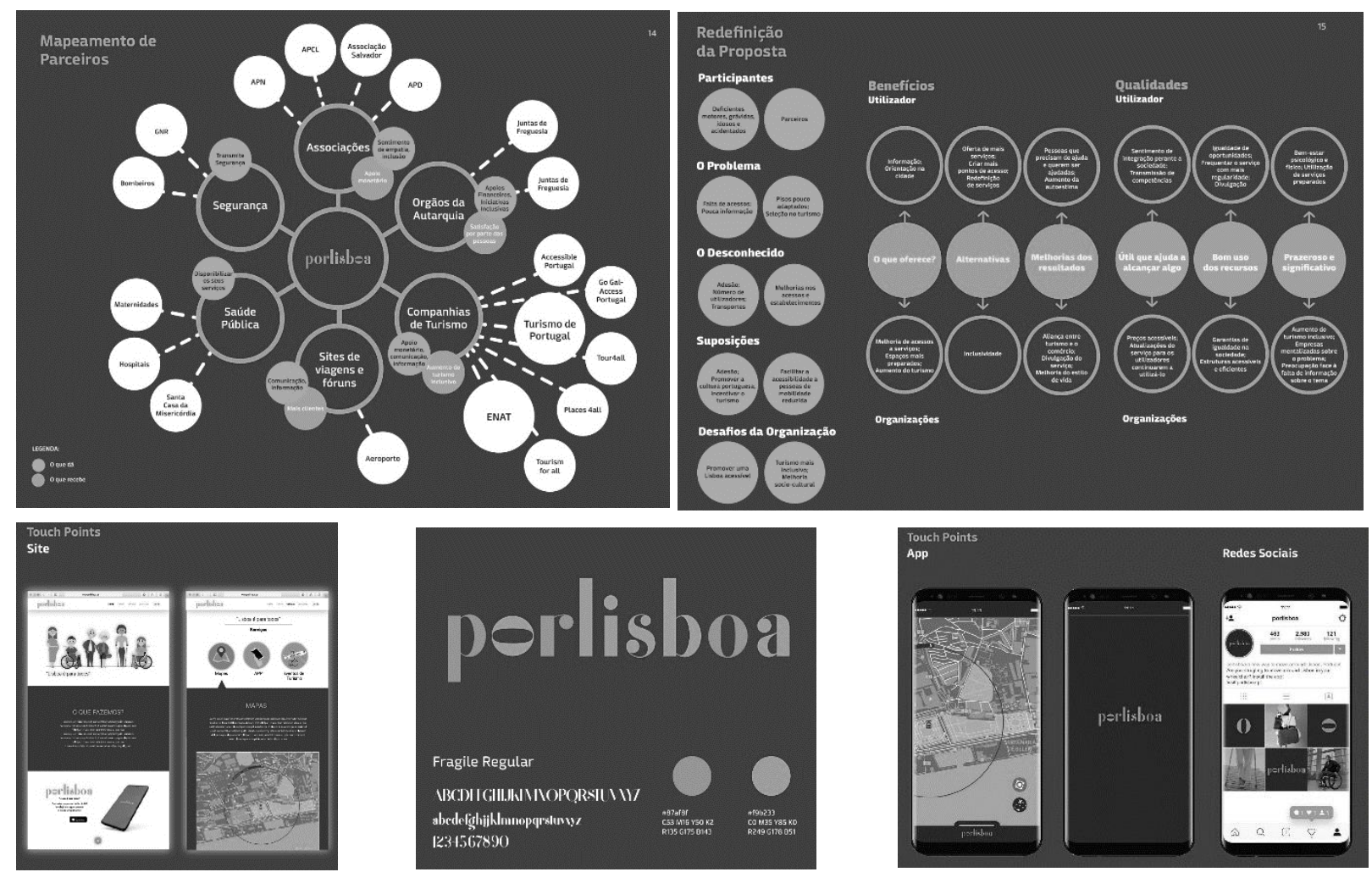

Figure 3. Group 10 -up-left side - partners constellation map; up-right side - the service proposition; down-left side- the site; down-middle figure - the logo symbol of the service, downright side - touchpoint- app

The use of all these methods (which results can be briefly appreciated in figures 2 and 3 ) depends heavily on the interaction with potential service users and other stakeholders. Being so, we had to guarantee a solid collaboration among students and inside teams as well as a fluid interaction with all the stakeholders that were vital to the services' creation. Furthermore, a final phase of the process - an iteration regarding the services' proposal was necessary to understand what needed to be changed and how to better monitor and ensure the post-service phase. This final stage also depended on interaction and collaboration with external elements.

\section{COLLABORATION AS THE NEURALGIC CENTER OF “DISTANT" DESIGNING}

Again, we highlight interaction and collaboration as being in the neuralgic centre of designing services in this specific context in which was mandatory to work online (at least during the 6 weeks of lockdown). As Bernardo and Duarte [6] observed "the screen mediated relationship severs the meaningfulness of a teacher-student relation." This aspect concerns what we call the $1^{\text {st }}$ dimension of collaboration - the one between teacher and students - that requests in this specific situation special care with the recognition of student' needs and concerns as well as a keen comprehension of student's environment (home) and responsibilities (e.g., caring), the access he/she has to communication resources and his/her level of proficiency using digital tools. The $2^{\text {nd }}$ dimension of collaboration is the one among students that are twofold:1) as a class group; 2) as a team. At this level, it was fundamental to make explicit the need for a strong connection not only among team members but especially among all the teams suggesting the use of a shared platform of information as well as the use of a class "WhatsApp" group to streamline the 
engagement in the designing process and to boost the joint comprehension and motivation to develop the tasks. Finally, the $3^{\text {rd }}$ dimension of collaboration gives respect to the interactions among students and stakeholders that must conquer the collaborative density. All these dimensions are translated in Figure 4 in which are visible all the dimensions connected in a system that presupposes fluxes among all of them and in both directions.

Figure 4. Collaboration as a neuralgic system of designing services.

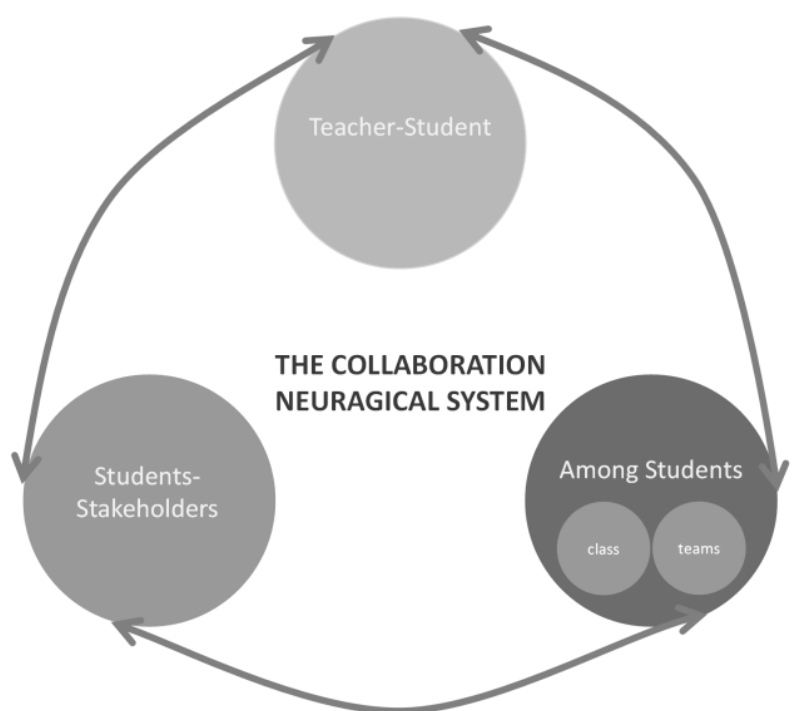

It is important to acknowledge that the openness to collaboration was reinforced during pandemics. That might be due to the fact (verbalized by several students) that all experienced the same constraint, being linked by that adversity.

\section{GUIDELINES TO COLLABORATE AT DISTANCE}

As a corollary of this teaching-learning experience in such atypical circumstances, it is now time to highlight some issues that are relevant to designing services in a distant mode and under mobility and physical interaction constraints.

\section{Regarding the Teacher's role}

1. Assume that collaborative mode as a neuralgic system that structures the process and that implies a clear commitment to the three dimensions of collaboration - 1. Teacher-Student; 2. A) Students in class; b) students as team members; 3 . Students-stakeholders.

2. Create enabler-moments at the beginning and end of the classes (10 minutes) dedicate to explicitly approach the existing and desired collaboration dynamics, energizing the class, "collect smiles" and explore the positive aspects of each constraint.

3. As a teacher make clear that you act as a "mobile member" of each group collaborating with your experience in each of the projects and activating a "cross-pollination" among groups.

4. Besides Synchronic classes teachers must create channels of communication with students that allow them to proceed its work outside classes - that includes the answer to doubts; unblock fixed moments in the process; assess preparatory work to be undertaken with stakeholder's etcetera.

\section{Regarding Students' intervention}

5. The collective feeling that "all are in the same boat", being united by this adversity must be assumed as a driver of the collaborative mode, boosting solidarity and meaningful interactions among students and amid teams.

6. The creation of groups based on the selection of team members according to their personalities and competencies enables richer contributions and stability among groups - since they present similar characteristics among their members.

7. The definition of roles inside the teams contributes to fewer misconceptions in the process opening space to clarification of decisions and prompting students to invest more in collaborative modes of sharing information, ideas, aiming to create a common language and a consistent and responsible proposal. 


\section{Stakeholders' engagement}

8. The collaboration to be created with stakeholders is favoured by this sentiment of "belonging to this larger group linked thru adversity" (that was the case of all stakeholders involved in the 13 projects). Being so students must recognize that openness and value it explicitly with stakeholders.

9. Since part of the target groups is segments of the population with less ability to react and develop resilience in a pandemic context cognitive ergonomics must be central in the development of solutions as well as in the engagement and interactions to be taken with these specific target groups.

10. "Making normal the unusual" must be the motto of the interactions i.e., students and teacher must facilitate the interaction moments with stakeholders finding ways of establishing comfortable inclusive and easy communication.

\section{CONCLUSIONS}

The lack of access to the fieldwork enabled resilient solutions such as phone interviews with elder people or zoom interviews with participants as well as presential encounters (after the lockdown period) that were bounded by pandemic rules. Furthermore, it had also an impact on the choices of the target groups to work with - students tend to choose convenient subjects of study. From the first moment, it was perceived that interactions were critical in this context, and collaboration popped up as a key driver of the projects' processes. It was remarkable how students quickly engaged in collaboration thru online tools complemented by enthusiastic share of information and thoughts in WhatsApp groups they immediately created. Equally rare was the willingness to collaborate on the part of stakeholders being clear that adversity favours human closeness, solidarity, and collaboration.

\section{ACKNOWLEDGEMENTS}

We acknowledge CIAUD, our Research Centre as well as the Foundation for Science and Technology (FCT) for funding this paper. Furthermore, recognition must be made to REDes - the research group that enabled us to support this type of research and reflections. Last but not least a special thanks to all the students and stakeholders involved in the work developed during this course.

\section{REFERENCES}

[1] Bozkurt A. and Sharma R. C. Emergency remote teaching in a time of global crisis due to Coronavirus pandemic, Asian Journal of Distance Education, 2020,15(1)

[2] Simonson M., Smaldino S. E. and Zvacek S. Teaching and learning at a distance: Foundations of distance education, 2015 (IAP-Information Age Publishing, Inc., Charlotte)

[3] Favilla K. and Pita T. When will fieldwork open up again? Beginning a project in pandemic times. Fennia, 2020, 198(1-2), 230-233.

[4] Hameed S., Badii A. and Cullen A. J. Effective e-learning integration with traditional learning in a blended learning environment. In EMCIS European and Mediterranean Conference on Information Systems. Dubai, UAE, 2008.

[5] Kimbell L. and Julier J. The Social Design Methods Menu, 2012 (Fieldstudio Ltd, London, United Kingdom.

[6] Bernardo N. and Duarte E. Design, Education, and the Online Tech-Pandemic. Strategic Design Research Journal, 2020, 13(3). 